\title{
Vigilancia tecnológica e inteligencia competitiva de un desarrollo tecnológico para la detección de Giardia, una innovación en salud
}

\author{
Emily Vargas, ${ }^{1}$ Sofía Duque Beltrán, ${ }^{2}$ Adriana Arévalo Jamaica ${ }^{2}$ y \\ Fabio Leonardo Quintero Vargas ${ }^{3}$
}

Forma de citar

Vargas E, Duque Beltrán S, Arévalo Jamaica A, Quintero Vargas FL. Vigilancia tecnológica e inteligencia competitiva de un desarrollo tecnológico para la detección de Giardia, una innovación en salud. Rev Panam Salud Publica. 2018;42:e82. https:/ / doi.org/10.26633/RPSP.2018.82

RESUMEN La giardiasis es una enfermedad causada por Giardia duodenalis, protozoario enteropatógeno que también es responsable de la enfermedad diarreica aguda y la gastroenteritis en humanos. Dadas las características de excreción intermitente propias de este parásito, la probabilidad de obtener resultados falsos negativos durante el proceso de diagnóstico es alta. En el Grupo de Parasitología del Instituto Nacional de Salud de Colombia se identificaron proteínas inmunogénicas presentes en quistes y trofozoítos del parásito en aislamientos colombianos de Giardia con potencial comercial y posibilidad de ser patentadas. Se realizó un estudio exploratorio descriptivo a partir de las características de la técnica en estudio, su estado de desarrollo y la viabilidad comercial de la invención. Se encontraron 390 patentes y se seleccionaron cinco invenciones similares que no representaban ningún riesgo frente a una eventual solicitud de patente. De los 36 estuches para el diagnóstico de Giardia disponibles en el mercado y las 21 licencias sanitarias otorgadas hasta diciembre del 2017, sólo cuatro y tres, respectivamente, proponen técnicas similares a la de la estudiada, pero ninguna de ellas cuenta con un registro de patente relacionado. La técnica en desarrollo es innovadora y puede aplicarse en el campo de la salud pública en la Región de las Américas con beneficios previsibles.

Palabras clave Vigilancia de productos comercializados; investigación; innovación; giardiasis; desarrollo tecnológico; Colombia.

La giardiasis es una enfermedad causada por Giardia duodenalis (snm. G. lamblia, G. intestinalis), un protozoario

\footnotetext{
1 EVidence Knowledge Brokering in Public Health SAS, Bogotá D.C., Colombia.

2 Grupo de Parasitología, Subdirección de Investigación Científica y Tecnológica, Dirección de Investigación en Salud Pública, Instituto Nacional de Salud, Bogotá D.C., Colombia.
}

enteropatógeno ubicuo que causa la enfermedad diarreica aguda y la gastroenteritis en humanos y sobre todo en niños

\footnotetext{
Subdirección de Innovación en Salud Pública, Dirección de Investigación en Salud Pública, Instituto Nacional de Salud, Bogotá D.C., Colombia. La correspondencia se debe dirigir a Fabio Leonardo Quintero Vargas, fquintero@ins. gov.co
}

$(1,2)$. El parásito puede transmitirse a través del agua tratada debido a su resistencia a los agentes de inactivación $(3,4)$, así como de los alimentos contaminados, de persona a persona o de animal a persona por vía fecal-oral $(4,5)$. La infección puede ser asintomática o manifestarse como diarrea persistente y síndrome de malabsorción (6) y, en casos crónicos,

Este es un artículo de acceso abierto distribuido bajo los términos de la licencia Creative Commons Attribution-NonCommercial-NoDerivs 3.0 IGO, que permite su uso, distribución y reproducción en cualquier medio, siempre que el trabajo original se cite de la manera adecuada. No se permiten modificaciones a los artículos ni su uso comercial. Al reproducir un artículo no debe haber ningún indicio de que la OPS o el artículo avalan a una organización o un producto específico. El uso del logo de la OPS no está permitido. Esta leyenda debe conservarse, junto con la URL original del artículo. 
producir retraso en el crecimiento y en el desarrollo cognitivo infantil (7).

La prevalencia de Giardia es más elevada en poblaciones segregadas con poco acceso a los servicios de salud. En el mundo, la prevalencia varía entre menos de $1 \%$ y más de $50 \%$ dependiendo del nivel de marginación y desigualdad (8). En África, Asia y Latinoamérica, por ejemplo, se registran más de 200 millones de casos anuales (9). En la encuesta de parasitismo intestinal realizada en Colombia en 2015 se notificó una prevalencia de giardiasis de $15,4 \%$ en la población escolar (10).

El género Giardia es un complejo de especies cuya morfología presenta pocas variaciones (1). Hasta la fecha, se han identificado ocho genotipos de Giardia (identificados de la A a la $\mathrm{H}$ ), pero sólo los genotipos A (AI, AII) y B (BIII, BIV) han demostrado ser patógenos en humanos $(1,2,11)$. Los avances en la clasificación del parásito no han tenido mayor influencia en su detección, que se realiza en muestras de materia fecal (única muestra o seriada). Dadas las características de excreción intermitente propias del parásito, la probabilidad de registrar falsos negativos es alta y, por ello, es más eficaz utilizar métodos invasivos como el aspirado duodenal, la impronta o la biopsia de intestino delgado (12), aunque son incómodos y costosos. Recientemente, se ha introducido la reacción en cadena de la polimerasa (PCR) en tiempo real, que requiere disponer de la tecnología apropiada y de recursos humanos capacitados, lo que dificulta su uso en zonas rurales o marginadas (13).

El Grupo de Parasitología del Instituto Nacional de Salud de Colombia (INS) identificó en aislamientos colombianos de Giardia (14) conservados en nitrógeno líquido $\left(-196{ }^{\circ} \mathrm{C}\right)$ proteínas en quistes y trofozoítos del parásito asociadas con antígenos de excreción, secreción, e inducción a la enfermedad (15). Mediante análisis in silico, se identificaron dos antígenos blanco (una proteína recombinante de 27,5 kDa, etiquetada como PR1, y otra de 34,2 kDa, etiquetada como PR2), y mediante espectrometría de masas, un antígeno (de $>116 \mathrm{kDa}$, etiquetado como PR3), que se clonaron para desarrollar tres antígenos recombinantes específicos de Giardia reconocidos mediante Western Blot por los anticuerpos policlonales IgG (desarrollados en conejo) e IgY anti-Giardia (desarrollados en gallina). (Por buenas prácticas en protección de la propiedad intelectual, el nombre de las proteínas no puede ser revelado. A los efectos de este artículo, se codificaron de la siguiente manera: Proteína recombinante $1(27,5 \mathrm{kDa})=$ "PR1", Proteína recombinante $2(34,2 \mathrm{kDa})=$ "PR2", Proteína recombinante 3 (de $>116 \mathrm{kDa}=$ "PR3".)

Los antígenos recombinantes se ensamblaron mediante una prueba ELISA indirecta de tipo sandwich (sensibilidad y especificidad en laboratorio de $100 \%$ ) para detectar el parásito en las heces. El tiempo de ejecución en campo fue de tres horas y media y no se detectó reacción cruzada con los microrganismos presentes en la materia fecal de humanos.

Dada la innovación que el procedimiento representaba, el Grupo obtuvo financiamiento para desarrollar la prueba de concepto (proof of concept, en inglés), la investigación aplicada y el desarrollo experimental, con el objetivo de validar el potencial de aplicación de los resultados.

Para tal fin, se desarrollaron los procesos de vigilancia tecnológica e inteligencia competitiva orientados a captar, seleccionar, analizar, difundir y comunicar información en ciencia y tecnología, tanto del exterior como de la propia organización, a fin de convertirla en conocimiento útil en la toma de decisiones (16) y en una herramienta esencial para detectar oportunidades de innovación tecnológica y nuevas ideas que faciliten la mejora de procesos y el desarrollo de productos y servicios en la organización $(17,18)$.

En este contexto, el objetivo principal de este estudio fue identificar y analizar el estado de desarrollo y la viabilidad comercial de esta técnica para la detección de Giardia.

\section{MATERIALES Y MÉTODOS}

Se hizo un estudio exploratorio descriptivo para conocer, mediante la consulta de fuentes secundarias, el estado de desarrollo y la viabilidad comercial de la técnica en estudio. Se analizaron las siguientes características de la técnica: el microorganismo objetivo, los estadios del parásito en los cuales hay detección, los antígenos objetivo, el método de obtención de los anticuerpos, el tipo de anticuerpos y de prueba diagnóstica, y el desarrollo del estuche de diagnóstico.

El estudio se desarrolló en cinco etapas determinadas según los criterios de los procesos de vigilancia tecnológica e inteligencia competitiva.
Etapa 1: determinación de las características de la técnica. La técnica se describió y se caracterizó según las siguientes variables: i) estadio del parásito que se debe detectar; ii) antígeno objetivo (proteína); iii) tipo de antígeno utilizado para el desarrollo de anticuerpos; iv) tipo de anticuerpos (monoclonales, policlonales); v) técnica utilizada para la detección; vi) sensibilidad y especificidad; vii) tiempo de ejecución de la prueba, y viii) reacción cruzada.

Etapa 2: definición de las estrategias de búsqueda. A partir de las variables definidas en la etapa 1, se seleccionaron las palabras que mayor espectro cubrían en cuanto a desarrollos existentes y equiparables a los de la técnica en estudio. La estrategia de búsqueda se enfocó en dos rutas de trabajo: i) el uso o identificación de las tres proteínas de interés en inmunodiagnóstico: la PR1 (27,5 kDa), la PR2 (34,2 kDa) y la PR3 (> $116 \mathrm{kDa})$, y ii) los métodos de obtención, prestando especial atención a las proteínas recombinantes. Los algoritmos resultantes se consultaron en la base de patentes PatBase ${ }^{\circledR}$ (19) sin límites de idioma o tiempo y en la de Scopus ${ }^{\circledR}$ sin límite de tiempo y en idioma inglés.

La ecuación de búsqueda específica de las tres proteínas de interés asociadas con Giardia fue la siguiente: Giardia AND ("recombinant methods" OR "protein expression" OR expression* OR production* OR "protein production" OR recombinant* OR "antigen production" OR obtain* OR getting OR sourcing OR extract* OR protein* OR antigen* OR "recombinant antigen") AND (PR1 OR PR2 OR PR3), en tanto que la ecuación de búsqueda general fue: Giardia AND ("recombinant methods" OR "protein expression" OR expression* OR production* OR "protein production" OR recombinant* OR "antigen production" OR obtain* OR getting OR sourcing OR extract* OR protein* OR antigen* OR "recombinant antigen").

Etapa 3: análisis de la novedad y del nivel inventivo de la técnica. Las variables escogidas en la etapa 1 se compararon frente a los desarrollos tecnológicos encontrados en la etapa 2. La lista de los registros de patentes y los artículos científicos recuperados en texto completo se depuró, y se seleccionaron y analizaron aquellos que guardaban mayor similitud con la técnica en estudio. 
Etapa 4: búsqueda de información comercial. Las variables o características técnicas de los productos comerciales usadas en el diagnóstico de Giardia se analizaron mediante una búsqueda en páginas web con las palabras "kit", "inmunodiagnóstico" y "Giardia".

Etapa 5: revisión de licencias sanitarias. Se revisaron las licencias sanitarias concedidas a productos comerciales en diferentes países de la Región de las Américas incluida Colombia. La información se obtuvo a partir de la revisión en la página web de la entidad competente de cada país: en Colombia, el Instituto Nacional de Vigilancia de Medicamentos y Alimentos (INVIMA); en Brasil, la Agencia Nacional de Vigilancia Sanitaria (ANVISA); en Ecuador, la Agencia Nacional de Regulación, Control y Vigilancia Sanitaria (ARCSA); en Panamá, la Dirección Nacional de Farmacia y Drogas; en Perú, la Dirección General de Medicamentos, Insumos y Drogas (DIGEMID); en Venezuela, el Instituto Nacional de Higiene Rafael Rangel, y en Bolivia, la Unidad de Medicamentos y Tecnologías en Salud (UNIMED).

\section{RESULTADOS}

\section{Estado de desarrollo de la técnica}

Análisis de patentes. Al aplicar las ecuaciones de búsqueda, se encontraron 390 patentes. Una vez analizados los documentos de patente asociados con la obtención de antígenos y anticuerpos o con la identificación de proteínas recombinantes de Giardia, se escogieron tres patentes para comparar su grado de similitud con las variables de la técnica en estudio (cuadro 1).

Las tres patentes son exclusivas para la detección de Giardia mediante inmunodiagnóstico cuando los antígenos recombinantes están presentes tanto en el quiste como en el trofozoíto del parásito. Cada una de las patentes se caracteriza por tener una proteína recombinante propia: la patente 1 (P1) ( $\delta$-giardina o $\beta$-giardina de $31 \mathrm{kDa})$, la patente 2 (P2) ( $\alpha$-1-giardina) y la patente 3 (P3) (GSA 65 de $65 \mathrm{kDa})$, ninguna de las cuales se emplea en la técnica en estudio. La obtención de anticuerpos en la P1 se hace a través de antígenos recombinantes con la producción de IgM. En la P2 la obtención de anticuerpos se logra a partir de antígenos recombinantes que dan como resultado anticuerpos policlonales GL.18. PC y monoclonales GL.5, en tanto que en la P3, los antígenos recombinantes dan como resultado anticuerpos policlonales monoespecíficos.

Entre las empresas solicitantes de patentes que ofrecen estuches diagnósticos para Giardia se encuentran Biosite Diagnostics, Coris Bioconcept y Savyon Diagnostics. Sus patentes están protegidas en los Estados Unidos de América, Australia, Canadá, México y Brasil (entre otros países latinoamericanos), así como en el marco del Tratado de Cooperación en Materia de Patentes (PCT) y la Oficina Europea de Patentes (EPO).

Análisis de publicaciones científicas. En la búsqueda bibliográfica se encontraron 420 artículos en la base de datos de Scopus $®$. Tras revisarlos, se seleccionaron cinco artículos cuyo texto completo se analizó en profundidad. En estos se describen desarrollos o tecnologías similares a la técnica en estudio (cuadro 2). Dos de los estudios se centran en la detección de Giardia en el suero humano, dos más recurren a procedimientos genómicos, y en otro se utiliza un anticuerpo monoclonal en materia fecal. En ninguno de los experimentos descritos se utilizó la combinación de las tres proteínas que son el objetivo de la técnica en estudio. Las técnicas de detección variaron según las proteínas identificadas e incluyeron Western Blot (IgG) o en doble dimensión, PCR y ELISA.

En el periodo de 2011 a 2012 hubo gran interés en la publicación de documentos científicos relacionados con la detección de Giardia. Los países con mayor investigación sobre este tema fueron los Estados Unidos de América, Australia, Brasil, Canadá y España. En Latinoamérica destacan la Universidad Nacional de Córdoba (Argentina) y el Centro de Investigación y de Estudios Avanzados del Instituto Politécnico Nacional (México).

\section{Viabilidad comercial de la invención}

Análisis de productos comerciales. Se analizaron 36 estuches comerciales para la detección de Giardia en humanos y animales en el mercado mundial. Destaca el hallazgo de cuatro que guardan mayor similitud con la técnica estudiada (cuadro 3), de los cuales tres son exclusivos para la detección de Giardia en heces humanas, en tanto que el otro (Giardial CryptosporidiumCHEK®) detecta Giardia y Cryptosporidium. En dos de los productos (Serazym ${ }^{\circledR}$ Giardia y Giardia $\mathrm{II}^{\mathrm{TM}}$ ) no se

\section{CUADRO 1. Patentes con mayor similitud a la técnica en estudio}

\begin{tabular}{|c|c|c|c|c|c|c|c|c|c|}
\hline \multirow[b]{2}{*}{ ID } & \multirow[b]{2}{*}{ Referencia } & \multicolumn{4}{|c|}{ Antígeno objetivo (proteínas) } & \multicolumn{2}{|c|}{ Desarrollo de anticuerpos } & \multicolumn{2}{|r|}{ Desarrollo del estuche } \\
\hline & & PR-1 & PR-2 & PR-3 & $\begin{array}{l}\text { Otras } \\
\text { proteínas }\end{array}$ & Antígeno que se utiliza & $\begin{array}{l}\text { Tipo de anticuerpo que se } \\
\text { obtiene (policlonal, } \\
\text { monoclonal) }\end{array}$ & Sí & No \\
\hline INS & Técnica en estudio & $X$ & $X$ & $X$ & No & Antígeno recombinante & Policlonal IgG e IgY & & $\begin{array}{l}\text { En desarrollo tecnológico } \\
\text { de prototipo }\end{array}$ \\
\hline P1 & $\begin{array}{l}\text { US 8,852,596 B1- } \\
2014(28)\end{array}$ & & & & $\begin{array}{l}\delta \text {-giardina y } \\
\text { ß-giardina } \\
(31 \mathrm{kDa})\end{array}$ & $\begin{array}{l}\text { Antígeno recombinante con la producción de } \\
\text { monoclonales de la línea celular de hibridoma } \\
5 \text { E11-19 (produce un anticuerpo monoclonal) }\end{array}$ & Monoclonal IgM & $X$ & \\
\hline P2 & $\begin{array}{l}\text { US 2003/0064417 } \\
\text { A1-2003 (29) }\end{array}$ & & & & $\alpha-1$-giardina & Antígeno recombinante & $\begin{array}{l}\text { Policlonal recombinante } \\
\text { GL.18.PC Monoclonal GL.5 }\end{array}$ & $X$ & \\
\hline P3 & $\begin{array}{l}\text { US5503983- } \\
1996(30)\end{array}$ & & & & $\begin{array}{l}\text { GSA } 65 \text {, peso } \\
\text { molecular de } \\
65 \mathrm{kDa}\end{array}$ & $\begin{array}{l}\text { Los antígenos se obtienen a partir del parásito } \\
\text { por ser de excreción/secreción y los } \\
\text { anticuerpos policlonales son monoespecíficos }\end{array}$ & Policlonal monoespecífico & & Pruebas de laboratorio \\
\hline
\end{tabular}

Fuente: elaboración propia a partir de datos de este estudio.

PR-1: proteína recombinante 1 - INS, PR-2: proteína recombinante 2 - INS y PR-2: proteína recombinante 3 - INS. P1, P2, P3: documentos de patente analizados. Todas las patentes han sido aprobadas, tienen como microorganismo objetivo Giardia y detectan el parásito en los dos estadios (trofozoíto y quiste). Fuente: referencia 36 
CUADRO 2. Artículos científicos sobre las tecnologías con mayor similitud con la técnica en estudio

\begin{tabular}{|c|c|c|c|c|c|c|}
\hline ID & Referencia & $\begin{array}{l}\text { Método de identificación de } \\
\text { la proteína }\end{array}$ & $\begin{array}{l}\text { ¿Utiliza las tres } \\
\text { proteínas de la } \\
\text { técnica del INS? }\end{array}$ & Proteínas utilizadas & $\begin{array}{c}\text { Técnicas } \\
\text { utilizadas para la } \\
\text { detección }\end{array}$ & $\begin{array}{l}\text { Posible } \\
\text { aplicabilidad }\end{array}$ \\
\hline$\overline{\mathrm{A} 1}$ & (31) & $\begin{array}{l}\text { Inmunoglobulinas } \\
\text { anti-Giardia en suero de } \\
\text { humano }\end{array}$ & No & $\begin{array}{l}\text { Proteínas de } 190,176,143,136,110,105,90,77,39,37,32, \\
27,22,21 \text { y } 15 \mathrm{kDa} \text {. Reactividad serológica para la respuesta } \\
\text { IgG anti-Giardia solamente en las proteínas de 190, 176, 143, } \\
51,30,23,17 \text { y } 15 \mathrm{kDa}\end{array}$ & $\begin{array}{l}\text { Western Blot } \\
(\operatorname{lgG})\end{array}$ & En experimento \\
\hline $\mathrm{A} 2$ & $(32)$ & Genómica & No & $\begin{array}{l}\text { Proteína recombinante nicotinamida / nicotinato } \\
\text { mononucleótido adenililtransferasa (GiNMNAT) }\end{array}$ & Inmunoblot & Inmunodiagnóstico \\
\hline A3 & (33) & Genómica & No & $\alpha$-giardinas de la 1 a 18 & PCR & $\begin{array}{l}\text { Para blanco } \\
\text { terapéutico }\end{array}$ \\
\hline A4 & (34) & $\begin{array}{l}\text { Inmunoglobulinas } \\
\text { anti-Giardia en suero de } \\
\text { humano }\end{array}$ & No & 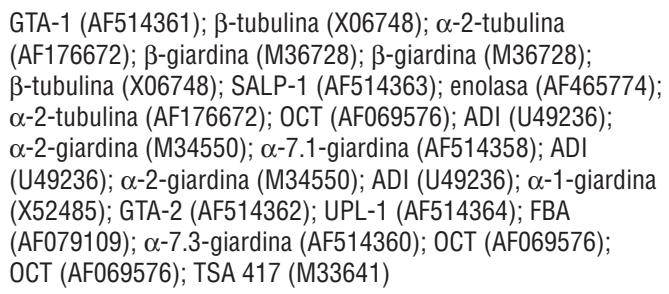 & $\begin{array}{l}\text { Western Blot en } \\
\text { doble dimensión }\end{array}$ & $\begin{array}{l}\text { Vacunas, y } \\
\text { herramientas } \\
\text { diagnósticas }\end{array}$ \\
\hline A5 & (35) & $\begin{array}{l}\text { Anticuerpo monoclonal en } \\
\text { materia fecal }\end{array}$ & No & CWP1 y CWP2 & ELISA & Inmunodiagnóstico \\
\hline
\end{tabular}

Fuente: elaboración propia a partir de datos de este estudio.

A1, A2, A3, A4, A5: artículos científicos analizados. Todos los artículos mencionaban Giardia como microorganismo objetivo. Fuente: referencia 36.

informa sobre el antígeno objetivo, y el antígeno utilizado (CWP-1) está presente tanto en el estadio de quiste como en el de trofozoíto. Serazym Giardia, PARATECT $^{\mathrm{TM}}$ y Giardia/Cryptosporidium CHEK® usan antígenos policlonales, pero solo los dos últimos se usan para captura y detección, lo cual se asemeja a la técnica en estudio. Sin embargo, ninguno de ellos utiliza anticuerpos policlonales IgG e IgY.

Los cuatro productos analizados se basan en la técnica de ELISA, especialmente la indirecta de tipo sandwich, cuya sensibilidad y especificidad son mayores de 85 y 95\%, respectivamente. El tiempo medio de ejecución de las pruebas es de dos horas.

Licencias sanitarias. Mediante la búsqueda en fuentes secundarias, se encontraron las licencias sanitarias que las entidades competentes de los países fronterizos con Colombia han concedido a diversos productos relacionados con la técnica en estudio. El número de licencias por país hasta abril de 2017 era de cinco en Colombia, tres en Bolivia, dos en Ecuador, diez en Brasil, y una en Perú. No se encontraron registros en Panamá ni en Venezuela.

Entre los hallazgos más relevantes cabe señalar que en ninguna de las licencias concedidas se menciona la combinación de los tres antígenos de la innovación que es motivo del presente estudio. Además, ninguno de los anticuerpos policlonales usados en los estuches comerciales es de origen aviar (IgY de captura), y aunque hay dos solicitudes de patentes relacionadas con la producción de IgY, no son procedimientos contra Giardia, un resultado que se complementó con una búsqueda específica con la ecuación "Giardia AND IgY", con la cual se encontraron 105 patentes de las cuales solo tres se relacionan con la tecnología en estudio. Sin embargo, en sus reivindicaciones, no se puso de manifiesto la protección de este tipo de anticuerpos contra Giardia.

\section{DISCUSIÓN}

La variabilidad genética de Giardia dificulta el inmunodiagnóstico, dado que las cepas de diferentes orígenes geográficos pueden presentar diferencias antigénicas $(14,20)$, que el parásito aprovecha para evadir el sistema inmunitario del huésped (21), una característica biológica del protozoo que justifica la búsqueda continua de herramientas inmunodiagnósticas más sensibles y específicas.

Aunque la tendencia de los métodos de detección se centra en la amplificación del ácido nucleico (PCR), esta técnica sigue requiriendo laboratorios que dispongan de los equipos y el personal idóneos (22). Dado que en muchas zonas de alto riesgo de infección por Giardia no existe la posibilidad de aplicar las pruebas moleculares y el transporte de muestras para el diagnóstico a centros especializados es costoso y poco oportuno, es necesario insistir en los métodos inmunodiagnósticos que pueden desarrollarse como pruebas rápidas de detección del parásito en el mismo sitio donde se toma la muestra, lo que contribuye, así, a reducir los costos del diagnóstico y a optimizar el tiempo de entrega del resultado y de administración del tratamiento $(22,23)$.

Por otro lado, el uso de la PCR en muestras fecales sigue siendo objeto de controversia (24) y en algunos estudios se recomienda tener cautela al analizar muestras de heces para la detectar G. duodenalis, ya que el ADN del parásito puede estar presente incluso después de que la infección se ha eliminado, lo cual produce resultados falsos positivos (13).

La técnica en estudio se basa en la combinación de tres antígenos recombinantes (PR1, PR2, PR3), que no se utilizan de forma simultánea en ninguna de las tecnologías detectadas. Solo uno de ellos, la PR1, se ha utilizado comercialmente como proteína inmunogénica.

Comparados con los anticuerpos monoclonales, los policlonales tienen la ventaja de que reconocen múltiples epítopes del antígeno blanco y se unen a epítopes inmunogénicos, aunque este hecho puede convertirse, en algunos casos, en una 


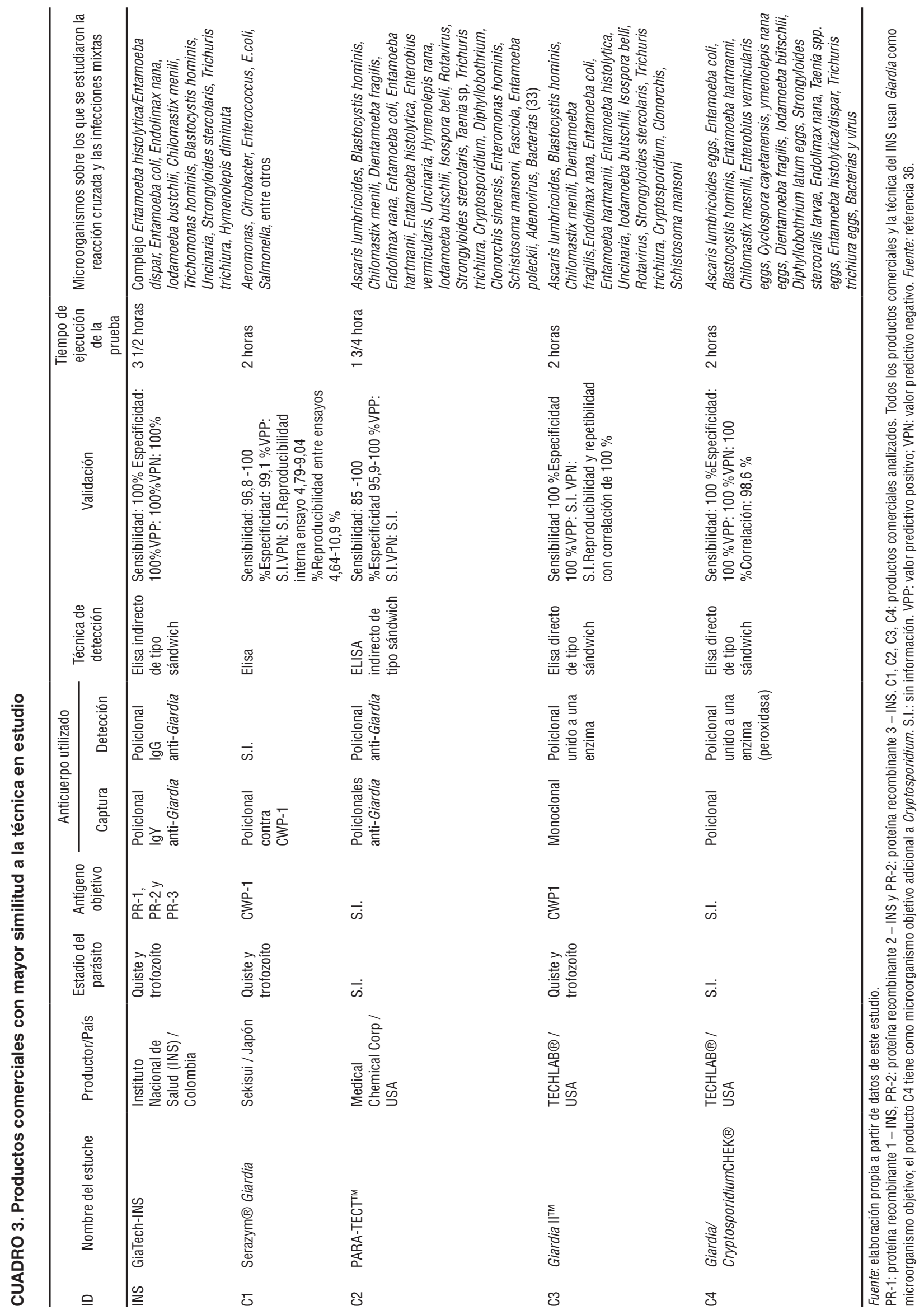


desventaja, porque podrían unirse a epítopes similares de otras proteínas y originar falsos positivos (25). Sin embargo, esta desventaja se evita al utilizar anticuerpos policlonales IgY, como lo hace la técnica en estudio.

Otra ventaja del uso de anticuerpos policlonales de captura de IgY antiGiardia se relaciona con las buenas prácticas de laboratorio. El uso de aves para obtener inmunoglobulinas por inmunización es un método menos complejo que el uso de mamíferos o roedores, en los cuales se requieren procesos invasivos que, en la mayoría de los casos, conducen a la muerte del animal (26). El uso de aves, además, disminuye las reacciones cruzadas, y aumenta la especificidad y la sensibilidad de la prueba por la distancia filogenética que existe entre ellas y los mamíferos (26).

Los retos que el desarrollo tecnológico impone en la optimización de las pruebas diagnósticas abren nuevas perspectivas para la técnica en estudio en el mercado biotecnológico. El Grupo de Parasitología del INS buscará incluir en la prueba otros patógenos (Cryptosporidium y Entamoeba histolytica patógena) causantes de infecciones intestinales en Colombia y en los países vecinos (10).

En cuanto al estado de la técnica en estudio, frente a los tres criterios establecidos para conceder una patente (grado de

1. Jerez-Puebla LE, Núñez FA, Pérez Santos L, Rojas Rivero L, Martínez Silva I, Ayllón Valdés L, et al. Molecular analysis of Giardia duodenalis isolates from symptomatic and asymptomatic children from La Habana, Cuba. Parasite Epidemiol. Control. 2017; 2(3):105-13. Disponible en https://doi. org/10.1016/j.parepi.2017.05.003 Acceso el 1 de abril de 2018.

2. Spotin A, Karamat M, Mahami-Oskouei M, Shahbazi A, Ahmadpour E, Mikaeili Galeh T, et al. Genetic variability and transcontinental sharing of Giardia duodenalis infrapopulations determined by glutamate dehydrogenase gene. Acta Trop. 2018;177: 146-56. Disponible en: https://doi.org/ 10.1016/j.actatropica.2017.10.001 Acceso el 2 de abril de 2018.

3. Zhang Y, Chen Z, An W, Xiao S, Yuan H, Zhang D, et al. Risk assessment of Giardia from a full scale MBR sewage treatment plant caused by membrane integrity failure. J Environ Sci (China). 2015;30:252-8. Disponible en: https://doi.org/10.1016/j. jes.2014.09.033 Acceso el 15 de julio de 2017.

4. Rousseau A, La Carbona S, Dumètre A, Robertson LJ, Gargala G, Escotte-Binet S, et al. Assessing viability and infectivity of foodborne and waterborne stages (cysts/

novedad, nivel inventivo y aplicación industrial), así como al riesgo de que existan otros desarrollos que puedan limitar o impedir la concesión de la patente, se pudo comprobar que la técnica en estudio no se ve afectada respecto a la novedad, especialmente por el uso conjunto de los tres antígenos recombinantes (PR1, PR2 y PR3), así como de anticuerpos policlonales de captura de IgY. Sobre el nivel inventivo, no se encontró ningún desarrollo con las mismas características de la técnica en estudio. Sin embargo, este podría verse afectado porque en los documentos se presentan algunas de las variables o características de la técnica en estudio de forma aislada. Respecto a la aplicación industrial, el riesgo es bajo, ya que la técnica propuesta puede emplearse como método diagnóstico en muestras ambientales y en suero de animales o de humanos.

Es importante indicar que la información recopilada en estudios de vigilancia tecnológica e inteligencia competitiva está sujeta a errores o tiene limitaciones inherentes a la información incluida en las bases de datos consultadas.

Los resultados obtenidos permiten concluir que la técnica en estudio se encuentra en la vanguardia de las pruebas inmunodiagnósticas de alta sensibilidad y especificidad, que pueden transferirse fácilmente a laboratorios de zonas rurales

\section{REFERENCIAS}

oocysts) of Giardia duodenalis, Cryptosporidium spp., and Toxoplasma gondii: a review of methods. Parasite. 2018;25:14. Disponible en https://doi.org/10.1051/parasite/2018009 Acceso el 12 de marzo de 2018.

5. Koehler AV, Jex AR, Haydon SR, Stevens MA, Gasser RB. Giardia/giardiasis - A perspective on diagnostic and analytical tools. Biotechnol Adv. 2014;32:280-9. Disponible en https://doi.org/10.1016/j.biotechadv.2013.10.009 Acceso el 10 de junio de 2017.

6. Ankarklev J, Jerlström-Hultqvist J, Ringqvist E, Troell K, Svärd SG. Behind the smile: cell biology and disease mechanisms of Giardia species. Nat Rev Microbiol. 2010;8:413-22. Disponible en https://doi. org/10.1038/nrmicro2317 Acceso el 10 de junio de 2017.

7. Carmona-Fonseca J, Correa-Botero A. Parásitos intestinales y desnutrición en niños en Urabá (Colombia) interpretados según las condiciones de vida del país: soledad y olvido. Rev Salud Ambient. 2013; 13:108-19.

8. Alum A, Sbai B, Asaad H, Rubino JR, Khalid Ijaz M. ECC-RT-PCR: a new method to determine the viability and infectivity of Giardia cysts. Int J Infect Dis. 2012;16: o con bajo desarrollo tecnológico, que, además, tiene un gran potencial de impacto en la salud pública en la Región, y que su grado de novedad y de nivel inventivo garantiza su reproducibilidad y la viabilidad de obtener una patente en la modalidad de invención.

Agradecimientos. Los autores agradecen al Departamento Administrativo de Ciencia, Tecnología e Innovación, Colciencias y al Instituto Nacional de Salud de Colombia, el financiamiento del proyecto, a la Corporación Tecnova, su apoyo como consultora externa, y a Ángela Liliana Albarracín Cárdenas y a Cesar Augusto Ramírez Segura su contribución en la ejecución del proyecto.

Conflictos de interés. Los autores declaran no tener conflictos de interés.

Financiación. Este proyecto (2104-70048773) fue financiado bajo el Contrato RC No 892-2015 Colciencias-INS-ACAC.

Declaración. Las opiniones expresadas por los autores son de su exclusiva responsabilidad y no reflejan necesariamente las opiniones de la Agencia de los Estados Unidos para el Desarrollo Internacional ni los criterios ni la política de la Organización Panamericana de la Salud o de la RPSP/PAJPH. e350-e353. Disponible en https://doi.org/ 10.1016/j.ijid.2012.01.004 Acceso el 10 de marzo de 2018.

9. Organización Mundial de la Salud. Modelo OMS de información sobre prescripción de medicamentos: medicamentos utilizados en las enfermedades parasitarias. $2^{a}$ ed: Protozoos: amibiasis y giardiasis. Ginebra: OMS; 1991:160.

10. Ministerio de Salud y Protección Social, Universidad de Antioquia. Encuesta nacional de parasitismo intestinal en población escolar 2012 - 2014. Medellín: Facultad Nacional Salud Pública, Universidad de Antioquia, El Ministerio; 2015.

11. García-Cervantes PC, Báez-Flores ME, Delgado-Vargas F, Ponce-Macotela M, Nawa Y, De-la-Cruz MC, et al. Giardia duodenalis genotypes among schoolchildren and their families and pets in urban and rural areas of Sinaloa, México. J Infect Dev Countries. 2017;11(2):180-87.

12. Goka AKJ, Rolston DDK, Mathan VI, Farthing MJG. The relative merits of faecal and duodenal juice microscopy in the diagnosis of giardiasis. Trans R Soc Trop Med Hyg. 1990;84(1):66-7.

13. Gotfred-Rasmussen H, Lund M, Enemark HL, Erlandsen M, Petersen E. Comparison 
of sensitivity and specificity of 4 methods for detection of Giardia duodenalis in feces: immunofluorescence and PCR are superior to microscopy of concentrated iodine-stained samples. Diagn Microbiol Infect Dis. 2016;84(3):187-90. Disponible en https://doi.org/10.1016/j.diagmicrobio.2015.11.005 Acceso el 1 de abril de 2018.

14. Arévalo A, Ravid Z, Duque S, Arévalo A, Nicholls RS. Genetic diversity of Giardia intestinalis populations in Colombia. Biomédica. 2007;27:34-41.

15. Ma'ayeh SY, Liu J, Peirasmaki D, HoÈrnaeus K, BergstroEmm Lind S, Grabherr $\mathrm{M}$, et al. Characterization of the Giardia intestinalis secretome during interaction with human intestinal epithelial cells: The impact on host. PLoS Negl Trop Dis. 2017; 11(12):e0006120. Disponible en https: / / doi. org/10.1371/journal.pntd.0006120 Acceso el 2 de abril de 2018.

16. Asociación Española de Normalización y Certificación. Norma 166000:2006. Gestión de la $\mathrm{I}+\mathrm{D}+\mathrm{i}$ : terminología y definiciones de las actividades de I+D+i. Madrid: Asociación Española de Normalización y Certificación; 2006:12.

17. Observatorio Virtual de Transferencia de Tecnología. Conceptos útiles en vigilancia tecnológica. Alicante: Universidad de Alicante, Banco Santander; 2014. Disponible en: https://www.ovtt.org/vigilancia-tecnologica-conceptos. Acceso el 20 de marzo de 2018.

18. Asociación Española de Normalización y Certificación. Norma UNE 166006:2011 Gestión de la I+D+i: Sistema de Vigilancia Tecnológica e Inteligencia Competitiva. Madrid: Asociación Española de Normalización y Certificación; 2011:17.

19. Minesoft Ltd., RWS Information. PatBase. Disponible en: https:/ / www.patbase.com/ login.asp. Acceso el 20 de marzo de 2018

20. Durigan M, Abreu AG, Zucchi MI, Franco RMB, de Souza AP. Genetic Diversity of Giardia duodenalis: Multilocus Genotyping Reveals Zoonotic Potential between Clinical and Environmental Sources in a Metropolitan Region of Brazil. PLoS One. 2014;9:e115489.

21. Prucca CG, Lujan HD. Antigenic variation in Giardia lamblia. Cell Microbiol. 2009;11: 1706-15. Disponible en https://doi.org/ 10.1111/j.1462-5822.2009.01367 Acceso el 2 de abril de 2018 .
22. de Fuentes I. Aplicación de técnicas rápidas y de biología molecular en la detección de parásitos en heces. XVI Congreso SEIMC Sociedad Española de Enfermedades Infecciosas y Microbiología Clínica, Bilbao, España; Sociedad Española de Enfermedades Infecciosas y Microbiología Clínica; 2012.

23. Álvis-Guzmán N, Orozco-Africano J, Paternina-Caicedo A, Coronell-Rodríguez W, Alvis-Estrada L, Jervis-Jálabe D, et al. Treatment costs of diarrheal disease and allcause pneumonia among children under-5 years of age in Colombia. Vaccine. 2013; 31(S3):C58-62. Disponible en https://doi. org/10.1016/j.vaccine.2013.05.035 Acceso el 3 de abril de 2018.

24. Ramírez JD, Heredia RD, Hernández C, León C, Moncada LI, Reyes $\mathrm{P}$, et al. Molecular diagnosis and genotype analysis of Giardia duodenalis in asymptomatic children from a rural area in central Colombia. Infect. Genet Evol. 2015;32: 208-13. Disponible en https://doi.org/ 10.1016/j.meegid.2015.03.015 Acceso el 30 de marzo de 2018

25. Mighell AJ, Hume WJ, Robinson PA. An overview of the complexities and subtleties of immunohistochemistry. Oral Dis. 1998;4(3):217-23.

26. García-Dabeiba A, Nicholls RS, Arévalo A Torres O, Duque S. Obtención, purificación y caracterización de anticuerpos policlonales IgY desarrollados en gallina, dirigidos contra aislamientos colombianos de Giardia duodenalis. Biomedica. 2005;25:451-63. Disponible en https://doi.org/10.7705/biomedica.v25i4.1372 Acceso el día 15 de julio de 2017.

27. Emery SJ, Lacey E, Haynes PA. Quantitative proteomics in Giardia duodenalis Achievements and challenges. Mol Biochem Parasitol. 2016;208(2):96-112. Disponible en https://doi.org/10.1016/ j.molbiopara.2016.07.002 Acceso el 2 de abril de 2018.

28. Jenkins MC, Fayer R, Santin M, Macarisin D, O'Brien C, inventors. The United States of America, as represented by the Secretary of Agriculture, assignee. Detection of Giardia lamblia trophozoites and cysts and protection against G. lamblia infection. U.S. Patent $N^{\circ}$ US 8,852,596 B1, October 7, 2014. Virginia: United States Patent and Trademarket Office; 2014.
29. Buechler J, Govindaraj S, Gray J, Valkirs GE, inventors. Biosite Diagnostics, assignee. Diagnostic assays for detection of Giardia lamblia. U.S. Patent $\mathrm{N}^{\circ}$ US 2003/0064417 A1, April 3, 2003. Virginia: United States Patent and Trademarket Office; 2003.

30. Rosoff JD, Stibbs HH, inventors. Alexon Biomedical, Inc., assignee. Method of diagnosis of giardiasis using Giardia lambliaspecific stool antigen. U.S. Patent $\mathrm{N}^{\circ}$ 5,503,983, April 2, 1996. Virginia: United States Patent and Trademarket Office; 1996

31. De Candia M, Pocaterra L, Rojas E, Hernán E, Hernán A, Jiménez JC. Respuesta de anticuerpos IgG contra fracciones de Giardia duodenalis en individuos infectados. Salus. 2016;20(2):13-7

32. Moreno-González PA, Díaz GJ, RamírezHernández MH. Producción y purificación de anticuerpos aviares (IgYs) a partir de cuerpos de inclusión de una proteína recombinante central en el metabolismo del NAD+. Rev Colomb Quim. 2013;42(2): 12-20.

33. Weiland MEL, McArthur AG, Morrison HG, Sogin ML, Svärd SG. Annexin-like alpha giardins: a new cytoskeletal gene family in Giardia lamblia. Int J Parasitol. 2005;35(6):617-26.

34. Daniel Palm JE, Weiland MEL, Griffiths WL, Ljungström I, Svärd SG. Identification of Immunoreactive Proteins during Acute Human Giardiasis. J Infect Dis. 2003, 187(12):1849-59.

35. Boone JH, Wilkins TD, Nash TE, Brandon JE, Macias EA, Jerris RC, et al. TechLab and Alexon Giardia enzyme-linked immunosorbent assay kits detect cyst wall protein 1. J Clin Microbiol. 1999;37(3):611-4

36. Instituto Nacional de Salud. Informe técnico: estado de la técnica "Desarrollo de antígenos recombinantes de Giardia para validar viabilidad de anticuerpos policlonales anti-Giardia y su utilidad inmunodiagnóstica". Bogotá D.C.: INS; 2017. (Informe técnico no publicado.)

Manuscrito recibido el 24 de agosto de 2017. Aceptado para publicación, tras revisión, el 13 de abril de 2018. 
ABSTRACT Giardiasis is a disease caused by Giardia duodenalis, an intestinal protozoan that causes acute diarrhea and gastroenteritis in humans. Given the characteristic intermittent excretion of this parasite, there is a high probability of false negative results during diagnosis. The Parasitology Group of Colombia's National Institute of Health identi-

Technological surveillance and competitive intelligence for a new technique to detect Giardia: an innovation in health

Keywords fied immunizing proteins present in the parasite's cysts and trophozoites in Colombian Giardia isolates with commercial and patentability potential. A descriptive exploratory study was conducted that focused on characteristics of the technique under study, its state of development, and the commercial viability of the invention. A total of 390 patents were found and five similar inventions were selected that did not pose any risk regarding an eventual patent application. Of the 36 Giardia diagnostic kits available on the market and the 21 health licenses granted as of December 2017, only four and three, respectively, offer techniques similar to the technique studied, but none of them has a related patent on record. The technique under development is innovative and can be applied in the field of public health in the Region of the Americas with foreseeable benefits.

Product surveillance; research; innovation; giardiasis; technological development; Colombia

RESUMO A giardíase é uma doença causada pela Giardia duodenais, protozoário enteropatogênico causador de doença diarreica aguda e gastroenterite em seres humanos. Como uma das características deste parasita é a excreção intermitente, a probabilidade de se obter resultados falso-negativos no processo diagnóstico é alta. O Grupo de Parasitologia do Instituto Nacional de Saúde da Colômbia identificou proteínas imunogênicas presentes em cistos e trofozoítos do parasita em isolados colombianos de Giardia com potencial comercial e possibilidade de patenteamento. Foi realizado um estudo exploratório descritivo das características da técnica em estudo, situação de desenvolvimento e viabilidade comercial da invenção. Foram encontradas 390 patentes e selecionadas cinco invenções similares sem risco de eventual pedido de patente. Dos 36 kits de diagnóstico de Giardia comercializados e 21 licenças sanitárias concedidas até dezembro de 2017, apenas quatro kits e três licenças de produtos empregam técnicas semelhantes à estudada, mas nenhum deles tem um registro de patente relacionado. A técnica em desenvolvimento é inovadora e pode ser empregada em saúde pública na Região das Américas com benefícios esperados.

Palavras-chave Vigilância de produtos comercializados; pesquisa; inovação; giardíase; desenvolvimento tecnológico; Colômbia 